\title{
MANEJ O EXITOSO DE LA HEMORRAGIA POSTPARTO POR ACREIISMO PLACENTARIO MEDIANTE TAMPONAMIENTO CON BALÓN DE BAKRI
}

\author{
Antonio Morales L. ${ }^{1}$, Alvaro Insunza F. ${ }^{1}$, Rodrigo Latorre R. ${ }^{1}$ \\ ${ }^{1}$ Hospital Padre Hurtado, Facultad de Medicina, Universidad del Desarrollo.
}

\section{RESUMEN}

Antecedentes: La hemorragia del postparto es una de las complicaciones de mayor morbimortalidad materna. Objetivo: Comunicar el uso exitoso del balón de Bakri en un caso de metrorragia del postparto. Metodología: Se presenta el dispositivo utilizado. Resultado: Control eficiente de la metrorragia postparto por acretismo placentario mediante el uso del balón de Bakri, que permitió conservar el útero. Conclusión: El balón de Bakri es una alternativa no quirúrgica para el control de la hemorragia del postparto.

\section{PALABRAS CLAVES: Hemorragia del post parto, balón de Bakri}

\section{SUMMARY}

Background: The postpartum haemorrhage is a complication with a higher maternal morbimortality. Objective: To communicate the successful use of Bakri balloon in the treatment of postpartum haemorrhage. Method: The use of the device is presented. Results: The use of Bakri balloon permitted to maintain the uterus in a case of postpartum haemorrhage due to placental accretism. Conclusion: The Bakri balloon is a non surgical alternative treatment for the postpartum haemorrhage.

\section{KEY WORDS: Postpartum haemorrhage, Bakri balloon}

\section{INTRODUCCIÓN}

La hemorragia postparto es una de las complicaciones más graves del puerperio inmediato y origina buena parte de la morbimortalidad materna (1). Clásicamente se describen como causas uterinas de la hemorragia postparto, los traumatismos (dehiscencias de cicatrices uterinas, roturas uterinas), la inercia y el sangrado del lecho placentario por anomalías de la placentación en todas sus variedades; éstas a su vez se asocian a anomalías de la inserción placentaria que aumentan en presencia de cicatrices uterinas (2). El tratamiento de la hemorragia post parto inmediata culmina mu- chas veces con la histerectomía e incluso, en algunas ocasiones, con ligaduras de las arterias hipogástricas, ambas con morbilidad importante $(3,4,5)$.

El objetivo de esta comunicación es informar el uso exitoso del balón de Bakri en un caso clínico de hemorragia del post parto por placenta acreta.

\section{CASO CLÍNICO}

Paciente de 36 años, multípara de 1, que es derivada desde el extrasistema cursando un embarazo de 36 semanas, para atención en el Hospital Padre Hurtado, con el diagnóstico de placenta 
previa oclusiva total, asintomática, pesquisada a las 35 semanas por ultrasonido. Se confirma el diagnóstico de ingreso observándose una placenta de inserción anterior, previa, oclusiva total y se programa cesárea electiva para las 37 semanas. La paciente es informada de las eventuales complicaciones y da su consentimiento para la cirugía. El hematocrito preoperatorio fue de $37,8 \%$ y hemoglobina de $12 \mathrm{~g} / \mathrm{dl}$. Se utilizó anestesia combinada y abordaje por laparotomía de Pfannestiel. Extracción fetal transplacentaria en podálica, sin contratiempos; se obtuvo un recién nacido de $3.130 \mathrm{~g}$, Apgar 9-10. Se procedió a efectuar alumbramiento manual al no lograr desprendimiento dirigido. Tras realizar legrado y escobillonaje de la cavidad uterina se advierte que en la zona de inserción placentaria persiste hemorragia profusa, sin restos placentarios visibles, con retracción uterina normal. El apoyo hemodinámico se realizó con 4 litros de cristaloides, 1 litro de coloides y drogas vasoactivas en bolo, lo que permitió estabilidad hemodinámica. En ese momento el hematocrito era de $23,3 \%$ y la hemoglobina de 7,4 $\mathrm{g} / \mathrm{dl}$, por lo que se inició transfusión inmediata de 2 unidades de glóbulos rojos. Por situación clínica descrita y persistencia de la metrorragia, se advierte a la paciente la indicación de histerectomía y se le propone intentar tratamiento conservador con balón de Bakri. Se instala el balón en forma retrógrada, a través de histerotomía; se realiza histerorrafia con catgut crómico $\mathrm{N}^{\circ} 1$ punto corrido y 2 ángulos. Se instilan 500 cc de solución fisiológica al balón. Con tacto vaginal se constata que el balón se encuentra mal posicionado, protruyendo a través del cérvix. Se abre la histerorrafia, vaciando previamente el balón y reinstalándolo correctamente. Se resutura la histerotomía, luego de lo cual se distiende el balón con $500 \mathrm{cc}$ de suero fisiológico. Se aprecia que una vez lleno el balón, el segmento mantiene una consistencia firme. No hay sangrado a través de la sutura y el flujo hemático a través de la sonda del balón es escaso. La paciente permanece estable y se continúa la cirugía. Al término de ésta la paciente mantiene la estabilidad hemodinámica y el sangrado por sonda es mínimo. Se indican retractores uterinos y antibióticos por 24 horas. La paciente evoluciona favorablemente, sin compromiso hemodinámico y la metrorragia visualizada por la sonda uterina disminuye progresivamente. El balón se retira a las 24 horas del procedimiento, disminuyendo la altura uterina de 24 a $17 \mathrm{~cm}$, sin metrorragia posterior a la extracción del dispositivo. Alta al tercer día post quirúrgico en buenas condiciones, con su recién nacido. Hematocrito al alta de $27,5 \%$ y hemoglobina de $8,8 \mathrm{~g} / \mathrm{dl}$. El control al $11^{\circ}$ día comprueba paciente en buenas condiciones, con útero bien retraído y sin metrorragia.

\section{DISCUSIÓN}

Una de las causas de la hemorragia del post parto inmediato es debida a acretismo placentario, el que se describe en $2 / 3$ de los casos de placenta previa. En casos de placenta previa con cicatriz de cesárea anterior, el riesgo de acretismo es directamente proporcional al número de cicatrices, llegando hasta $67 \%$ con el antecedente de 3 cesáreas previas (6). La verdadera incidencia de acretismo placentario es difícil de determinar dado que el diagnóstico de certeza requiere del estudio anatomopatológico del útero. Sin embargo, éste se puede realizar sólo en los casos de placenta increta, percreta y de acretismo local que requirió histerectomía. Sólo hay datos aislados de pacientes que se manejaron médicamente en casos de diagnóstico clínico de acretismo local.

El manejo conservador ofrecido hasta ahora a éstas pacientes varía ampliamente y consiste en uso de retractores uterinos, ligadura de arterias uterinas y/o hipogástricas, suturas al lecho placentario, embolización de arterias uterinas y uso de métodos mecánicos de compresión como packing de compresas y dispositivos intrauterinos como la sonda Sengstaken-Blakemore utilizada tradicionalmente en hemorragia de origen esofágico (7). En los últimos años se ha reportado el uso de tamponamiento intrauterino con un balón especialmente diseñado para ello, creado por Bakri (SOS Bakri Tamponade Balloon-Cook ${ }^{\circledR}$ ) específicamente para la hemorragia de causa uterina, con resultados promisorios. Este dispositivo, hecho de silicona, está constituido por una sonda tubular de $24 \mathrm{Fr}$. de diámetro y $58 \mathrm{~cm}$ de largo; en un extremo presenta un balón con capacidad de hasta $800 \mathrm{ml}$ y la sonda de drenaje fenestrada; en el otro cabo presenta el orificio de drenaje que se conecta a un colector urinario estándar para la salida de los loquios y una llave de tres pasos para el llenado del balón (Figura 1) (8).

El protocolo de uso sugerido en los reportes, propone la instalación del balón como terapia inmediatamente antecesora a la decisión de histerectomía. Llenado con 500 a 800 cc de suero fisiológico y la asociación de retractores uterinos, más la observación estricta de los parámetros hemodinámicos y de la cantidad de flujo a través de la sonda fenestrada del balón. El balón se 


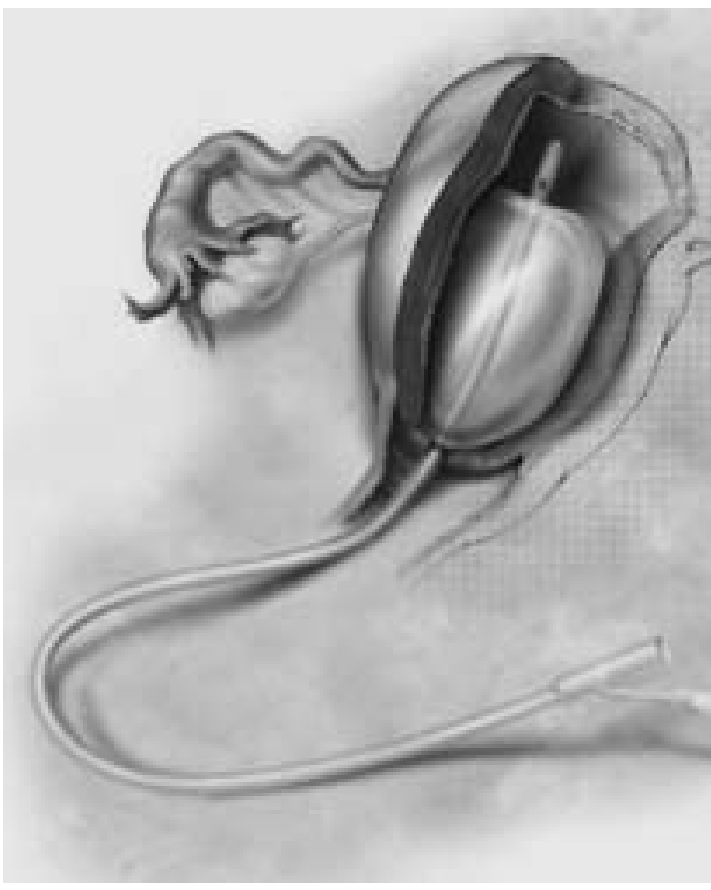

Figura 1. Balón de Bakri en posición intrauterina.

puede colocar por vía anterógrada desde la vagina o por vía retrógrada a través de una histerotomía. Asimismo, se sugiere el uso de un tamponamiento vaginal con compresas para evitar la salida del balón si el cuello está dilatado (9).

En Chile, la muerte materna por hemorragia del postparto ocupa el 5o lugar de causalidad, con 44 muertes maternas en el período 19902000 (10). Esta grave complicación ha motivado que en los últimos dos Congresos de la Sociedad Chilena de Obstetricia y Ginecología se presenten trabajos relacionados con histerectomía obstétrica y ligadura de arterias hipogástricas, siendo en ellos la hemorragia del postparto por anomalías de la inserción placentaria la principal indicación, y en los que se comenta las importantes complicaciones de ambas terapias $(3,4,11,12)$. El uso de tamponamiento intrauterino con compresas o gasas generalmente no resuelve el sangrado y solo posterga la histerectomía, muchas veces hay agravamiento de la condición hemodinámica de la paciente. La ventaja de la técnica de tamponamiento por balón es la compresión efectiva del lecho placentario sangrante, tal como lo observamos en el caso que comentamos, con respuesta hemodinámica inmediata que permitió evitar la histerectomía y sin morbilidad post operatoria. Es destacable la fácil instalación del balón de Bakri y la posibilidad que otorga para monitorear la cuantía del flujo uterino.

B-Lynch y cols (13) en 1997, reportaron una técnica quirúrgica ideada para preservar la fertilidad futura de la paciente, con menor complejidad técnica que la ligadura de las arterias hipogástricas. Recientemente, Mitelman y cols (14) comunicaron una interesante y original alternativa quirúrgica para el control de la metrorragia grave del puerperio con conservación del útero.

Estimamos que el uso del balón intrauterino es una alternativa promisoria y válida en el tratamiento de la hemorragia postparto por sangrado del lecho placentario, permitiendo con ello evitar cirugías de mayor envergadura, con el riesgo de morbilidad que éstas siempre conllevan y conservando el útero. No tenemos hasta ahora experiencia en el uso del balón de Bakri en su segunda indicación, cual es la hemorragia por inercia intratable.

\section{BIBLIOGRAFÍA}

1. Chichakli LO, Atrash HK, MacKay AP, Musani AS, Berg CJ. Pregnancy-related mortality in the United States due to hemorrhage: 1979-1992. Obstet Gynecol 1999; 94: 721-5.

2. American College of Obstetricians and Gynecologists. ACOG comittee opinion. Placenta accreta. Number 266, January 2002. Int J Gynaecol Obstet 2002; 77(1): 77-78.

3. Torres O, Buebo F, González R, Bronda A, Pinedo M. Ligadura de las arterias iliacas internas en cirugía obstétrica. C-44 Congreso SOCHOG 2001.

4. Latorre R, Levancini M, Pineda R, Yánez C, Cabellos A, Paiva E. Histerectomía obstétrica: Incidencia y características clínicas. C-54 Congreso SOCHOG 2001.

5. García-Huidobro M. Alumbramiento Patológico. En: García-Huidobro M, Hasbún J. Urgencias y Complicaciones en Obstetricia. Editorial Mediterráneo, 2006

6. Clark SL, Koomings PP, Pelan JP. Placenta previa/ accreta and previous cesarean section. Obstet Gynecol 1985; 66: 89-92.

7. Frenzel D, Condous GS, Papageorghiou AT, Mc Whiney NA. The use of the "tamponade test" to stop massive obstetric haemorrhage in placenta accreta BJOG 2005; 112: 676-77.

8. Bakri YN. Balloon device for control of obstetrical bleeding. Euro J Obstet Gynecol Repro Biol 1999; 86: S84.

9. Bakri YN, Mari A, Abdul Jabbar F. Tamponadeballoon for obstetrical bleeding. Int J Obstet 2001; 74(2): 139-42. 
10. Donoso E. Reducción de la mortalidad materna en Chile de 1990 a 2000. Rev Panam Salud Pública 2004; 15(5): 326-30.

11. Polanco M, Riveros R, Hernández A. Incidencia, causas y complicaciones de la histerectomía obstétrica. C-58 congreso SOCHOG 2005.

12. Theodor N, Valenzuela $P$, de Jourdan $F$, Bolte $L$, Merino P, Castiblanco A, Cuello M. Histerectomías obstétricas de emergencia. Experiencia Universidad Católica. C-58 congreso SOCHOG 2005.
13. B-Lynch C, Coker A, Lawal AH, Abu J, Cowen MJ. The B-Lynch surgical technique for the control of massive haemorrhage: an alternative to hysterectomy? Five reported. Br J Obstet Gynaecol 1997; 104(3); 372-5.

14. Mitelman G, La Rosa G, Martínez F, Cadima R. Alternativa quirúrgica en la hemorragia grave del puerperio. Rev Chil Obstet Ginecol 2004; 69(4): 316-18. 